\title{
A Subclass of Harmonic Functions Related to a Convolution Operator
}

\author{
Saqib Hussain, ${ }^{1}$ Akhter Rasheed, ${ }^{1}$ and Maslina Darus ${ }^{2}$ \\ ${ }^{1}$ Department of Mathematics, COMSATS Institute of Information Technology, Abbottabad Campus, Abbottabad, Pakistan \\ ${ }^{2}$ School of Mathematical Sciences, Faculty of Science and Technology, Universiti Kebangsaan Malaysia, 43600 Bangi, \\ Selangor, Malaysia
}

Correspondence should be addressed to Saqib Hussain; saqib_math@yahoo.com

Received 23 May 2016; Accepted 21 July 2016

Academic Editor: Wilfredo Urbina

Copyright (C) 2016 Saqib Hussain et al. This is an open access article distributed under the Creative Commons Attribution License, which permits unrestricted use, distribution, and reproduction in any medium, provided the original work is properly cited.

We introduce a new subclass of harmonic functions by using a certain linear operator. For this class we derive coefficient bounds, extreme points, and inclusion results and also show that this class is closed under an integral operator.

\section{Introduction}

Harmonic functions have long been used in the representation of minimal surface; for example, Heinz [1] in 1952 used such mappings in the study of the Gaussian curvature of nonparametric minimal surface over the unit disc. Such mappings have vast application in the field of engineering, physics, electronics, medicine, operations research, aerodynamics, and other branches of applied mathematics (see [2]).

$f(z)=u(x, y)+i v(x, y)$ is said to be complex valued harmonic function if both $u$ and $v$ are continuous and real harmonic; that is, $u_{x x}+u_{y y}=0$ and $v_{x x}+v_{y y}=0$. In simply connected domain [3], we can write $f(z)=h(z)+\overline{g(z)}$, where $h$ and $g$ are analytic in $E=\{z:|z|<1\}$. We call $h$ the analytic part and $g$ the coanalytic part of $f$. The necessary and sufficient condition for a function $f(z)=h(z)+\overline{g(z)}$ to be univalent and sense preserving in $E$ is $\left|h^{\prime}(z)\right|>\left|g^{\prime}(z)\right|$ (see [3]). A function $f(z)=h(z)+\overline{g(z)}$ is in class $\delta_{\mathscr{H}}$ if it is harmonic, univalent, and sense preserving in $E$, where $h$ and $g$ have the following series:

$$
\begin{aligned}
& h(z)=z+\sum_{k=2}^{\infty} a_{k} z^{k}, \\
& g(z)=\sum_{k=1}^{\infty} b_{k} z^{k},
\end{aligned}
$$

Hence

$$
f(z)=z+\sum_{k=2}^{\infty} a_{k} z^{k}+\overline{\sum_{k=1}^{\infty} b_{k} z^{k}} \quad\left|b_{1}\right|<1 .
$$

Note that if the coanalytic part $g$ is identically zero, then $\mathcal{S}_{\mathscr{H}}$ reduces to well-known class of normalized univalent analytic functions $\delta$. For this class, the function $f(z)$ may be expressed as

$$
f(z)=z+\sum_{k=2}^{\infty} a_{k} z^{k}
$$

A function $f(z)$ given by (2) is said to be harmonic starlike of order $\beta(0 \leq \beta<1)$ for $|z|=r<1$, if

$$
\frac{\partial}{\partial \theta}\left(\arg \left(f\left(r e^{i \theta}\right)\right)\right)>\beta,
$$

or equivalently

$$
\operatorname{Re}\left(\frac{z h^{\prime}(z)-\overline{z g^{\prime}(z)}}{h(z)+g(z)}\right)>\beta, \quad z=r e^{i \theta} .
$$

The class of all harmonic starlike functions of order $\beta$ is denoted by $\mathcal{S}_{\mathscr{H}}^{*}(\beta)$. This class was studied by Jahangiri [4]. The cases $\beta=0$ and $b_{1}=0$ were studied by Silverman and Silvia [5] and Silverman [6]. 
For $F(z)=z+\sum_{k=2}^{\infty} A_{k} z^{k}+\sum_{k=1}^{\infty} B_{k} z^{k}$ and $f(z)$ given by (2), the convolution is denoted by $f * F$ and defined as

$$
f * F=z+\sum_{k=2}^{\infty} a_{k} A_{k} z^{k}+\sum_{k=1}^{\infty} b_{k} B_{k} z^{k} .
$$

Let $\alpha_{1}, A_{1}, \ldots, \alpha_{p}, A_{p}$ and $\beta_{1}, B_{1}, \ldots, \beta_{q}, B_{q}(p, q \in N=$ $\{1,2,3, \ldots\})$ be positive real parameter such that

$$
1+\sum_{k=1}^{\infty} B_{k}-\sum_{k=1}^{\infty} A_{k} \geq 0
$$

The Wright generalized hypergeometric function [7] is defined as

$$
\begin{aligned}
{ }_{p} \Psi_{q}\left[\left(\alpha_{1}, A_{1}\right), \ldots,\left(\alpha_{p}, A_{p}\right) ;\left(\beta_{1}, B_{1}\right), \ldots,\left(\beta_{q}, B_{q}\right) ; z\right] \\
={ }_{p} \Psi_{q}\left[\left(\alpha_{i}, A_{i}\right)_{1, p} ;\left(\beta_{i}, B_{i}\right)_{1, q} ; z\right]
\end{aligned}
$$

which is defined by

$$
\begin{aligned}
{ }_{p} \Psi_{q}\left[\left(\alpha_{i}, A_{i}\right)_{1, p} ;\left(\beta_{i}, B_{i}\right)_{1, q} ; z\right] \\
=\sum_{k=0}^{\infty} \frac{\Pi_{i=1}^{p} \Gamma\left(\alpha_{i}+k A_{i}\right)}{\Pi_{i=1}^{q} \Gamma\left(\beta_{i}+k B_{i}\right)} \frac{z^{k}}{k !} \quad z \in E .
\end{aligned}
$$

If $A_{i}=1(i=1,2, \ldots, p)$ and $B_{i}=1(i=1,2, \ldots, q)$, we have the relationship

$$
\begin{aligned}
\Omega_{p} \Psi_{q}\left[\left(\alpha_{i}, A_{i}\right)_{1, p} ;\left(\beta_{i}, B_{i}\right)_{1, q} ; z\right] \\
={ }_{p} F_{q}\left(\alpha_{1}, \ldots, \alpha_{p} ; \beta_{1}, \ldots, \beta_{q} ; z\right) \\
\quad=\sum_{k=0}^{\infty} \frac{\left(\alpha_{1}\right)_{k} \cdots\left(\alpha_{p}\right)_{k}}{\left(\beta_{1}\right)_{k} \cdots\left(\beta_{q}\right)_{k}} \frac{z^{k}}{k !} .
\end{aligned}
$$

$\left(p \leq q+1 ; p, q \in N_{0}=N \cup\{0\} ; z \in E\right)$ is the generalized hypergeometric function [8], where $N$ denotes the set of all positive integers and $(\alpha)_{k}$ is the Pochhammer symbol and

$$
\Omega=\frac{\Pi_{i=1}^{p} \Gamma\left(\alpha_{i}\right)}{\Pi_{i=1}^{q} \Gamma\left(\beta_{i}\right)} .
$$

Dziok and Srivastava [8] introduced a linear operator which is generalization of Dziok-Srivastava operator [8-10], Carlson-Shaffer operator [11], and the generalized Bernardi's integral operator [12].

Dziok and Raina [13] considered the linear operator $W\left[\left(\alpha_{i}, A_{i}\right)_{1, p} ;\left(\beta_{i}, B_{i}\right)_{1, q}\right]: \mathcal{S} \rightarrow \mathcal{S}$ defined by

$$
\begin{aligned}
W & {\left[\left(\alpha_{i}, A_{i}\right)_{1, p} ;\left(\beta_{i}, B_{i}\right)_{1, q}\right] f(z) } \\
& ={ }_{p} \Phi_{q}\left[\left(\alpha_{i}, A_{i}\right)_{1, p} ;\left(\beta_{i}, B_{i}\right)_{1, q} ; z\right] * f(z),
\end{aligned}
$$

where ${ }_{p} \Phi_{q}\left[\left(\alpha_{i}, A_{i}\right)_{1, p} ;\left(\beta_{i}, B_{i}\right)_{1, q} ; z\right]$ is given by

$$
\begin{aligned}
& { }_{p} \Phi_{q}\left[\left(\alpha_{i}, A_{i}\right)_{1, p} ;\left(\beta_{i}, B_{i}\right)_{1, q} ; z\right] \\
& \quad=\Omega z_{p} \Psi_{q}\left[\left(\alpha_{i}, A_{i}\right)_{1, p} ;\left(\beta_{i}, B_{i}\right)_{1, q} ; z\right] .
\end{aligned}
$$

For $f(z) \in \mathcal{S}$, we have

$$
\begin{gathered}
W\left[\left(\alpha_{i}, A_{i}\right)_{1, p} ;\left(\beta_{i}, B_{i}\right)_{1, q}\right] f(z) \\
=z+\sum_{k=2}^{\infty} \Omega \sigma_{k}\left(\alpha_{1}\right) a_{k} z^{k}
\end{gathered}
$$

where $\sigma_{k}\left(\alpha_{1}\right)$ is given by

$$
\begin{aligned}
& \sigma_{k}\left(\alpha_{1}\right) \\
& =\frac{\Gamma\left(\alpha_{1}+A_{1}(k-1)\right) \cdots \Gamma\left(\alpha_{p}+A_{p}(k-1)\right)}{\Gamma\left(\beta_{1}+\beta_{1}(k-1)\right) \cdots \Gamma\left(\beta_{q}+\beta_{q}(k-1)\right)(k-1) !} .
\end{aligned}
$$
$W\left[\alpha_{1}\right]$

For our convenience we write $W\left[\left(\alpha_{i}, A_{i}\right)_{1, p} ;\left(\beta_{i}, B_{i}\right)_{1, q}\right]=$

Motivated by the work of $[4,14-21]$, we extend the work of Chandrashekar et al. [22] by introducing some new subclasses of $\mathcal{S}_{\mathscr{H}}$ using the generalized hypergeometric function.

Definition 1. A function $f \in \mathcal{S}_{\mathscr{H}}$ is in class $\mathscr{G}_{\mathscr{H}}\left(\left[\alpha_{1}, A_{1}\right.\right.$, $\left.\left.\beta_{1}, B_{1}\right], \gamma\right)$ if

$$
\operatorname{Re}\left\{1+\left(1+e^{i \phi}\right) \frac{\left[z^{2}\left(W\left[\alpha_{1}\right] h(z)\right)^{\prime \prime}+\overline{2\left(W\left[\alpha_{1}\right] g(z)\right)^{\prime}+z^{2}\left(W\left[\alpha_{1}\right] g(z)\right)^{\prime \prime}}\right]}{z\left(W\left[\alpha_{1}\right] h(z)\right)^{\prime}-\overline{z(W g(z))^{\prime}}}\right\} \geq \gamma
$$

Definition 2. Let $\mathscr{T}_{\mathscr{H}}$ denote the class of functions of the form

$$
f(z)=z-\sum_{k=2}^{\infty}\left|a_{k}\right| z^{k}-\overline{\sum_{k=1}^{\infty}\left|b_{k}\right| z^{k}}, \quad\left|b_{1}\right|<1,
$$

and $\mathscr{T}_{\mathscr{H}}\left(\left[\alpha_{1}, A_{1}, \beta_{1}, B_{1}\right], \gamma\right)=\mathscr{G}_{\mathscr{H}}\left(\left[\alpha_{1}, A_{1}, \beta_{1}, B_{1}\right], \gamma\right) \cap \mathscr{T}_{\mathscr{H}}$
Throughout this paper, we shall assume $0 \leq r<1, f=$ $h+\bar{g}, \Omega$, and $\sigma_{k}\left(\alpha_{1}\right)$ as given in (11) and (15), respectively, unless otherwise mentioned.

\section{Main Results}

In Theorem 3, we shall present a sufficient condition for $f \in$ $S_{H}$ to be in class $\mathscr{G}_{\mathscr{H}}\left(\left[\alpha_{1}, A_{1}, \beta_{1}, B_{1}\right], \gamma\right)$. 
Theorem 3. Let $f=h+\bar{g}$ be given by (2). If

$$
\sum_{k=1}^{\infty} k\left(\frac{(2 k-1-\gamma)}{1-\gamma}\left|a_{k}\right|+\frac{(2 k+1+\gamma)}{1-\gamma}\left|b_{k}\right|\right) \sigma_{k}\left(\alpha_{1}\right) \Omega
$$

Proof. When inequality (18) holds for the coefficients of $f=h+\bar{g}$ given in (2), we have to show that inequality (16) is satisfied. Arranging the left side inequality (16), we have

$\leq 2$,

then $f \in \mathscr{G}_{\mathscr{H}}\left(\left[\alpha_{1}, A_{1}, \beta_{1}, B_{1}\right], \gamma\right)$.

$$
\begin{aligned}
\operatorname{Re} & \left\{\frac{z\left(W\left[\alpha_{1}\right] h(z)\right)^{\prime}+\left(1+e^{i \phi}\right) z^{2}\left(W\left[\alpha_{1}\right] h(z)\right)^{\prime \prime}+\left(1+2 e^{i \phi}\right) \overline{z\left(W\left[\alpha_{1}\right] g(z)\right)^{\prime}}+\left(1+e^{i \phi}\right) \overline{z^{2}\left(W\left[\alpha_{1}\right] g(z)\right)^{\prime \prime}}}{z\left(W\left[\alpha_{1}\right] h(z)\right)^{\prime}-\overline{z\left(W\left[\alpha_{1}\right] g(z)\right)^{\prime}}}\right\} \geq \gamma \\
& =\operatorname{Re} \frac{A(z)}{B(z)} .
\end{aligned}
$$

As we know $\operatorname{Re}(w) \geq \gamma$ if and only if $|1-\gamma+w| \geq|1+\gamma-w|$, it is sufficient to show that

$$
|A(z)+(1-\gamma) B(z)|-|A(z)-(1+\gamma) B(z)| \geq 0 .
$$

Substituting the values $A(z)$ and $B(z)$ in left side of (20), we obtain

$$
\begin{aligned}
& |A(z)+(1-\gamma) B(z)|-|A(z)-(1+\gamma) B(z)| \geq(2 \\
& -\gamma)|z|-\sum_{k=2}^{\infty} k(2 k-\gamma)\left|a_{k}\right| \sigma_{k}\left(\alpha_{1}\right) \Omega|z|^{k} \\
& \quad-\sum_{k=1}^{\infty} k(2 k+\gamma)\left|b_{k}\right| \sigma_{k}\left(\alpha_{1}\right) \Omega|z|^{k}-\gamma|z| \\
& \quad-\sum_{k=2}^{\infty} k(2 k-2-\gamma)\left|a_{k}\right| \sigma_{k}\left(\alpha_{1}\right) \Omega|z|^{k} \\
& \quad-\sum_{k=1}^{\infty} k(2 k+2+\gamma)\left|a_{k}\right| \sigma_{k}\left(\alpha_{1}\right) \Omega|z|^{k} \geq 2(1-\gamma) \\
& \quad+\left\{1-\sum_{k=2}^{\infty} k \frac{(2 k-1-\gamma)}{1-\gamma}\left|a_{k}\right|\left|\sigma_{k}\left(\alpha_{1}\right)\right||\Omega|\right. \\
& \left.\quad+\sum_{k=1}^{\infty} k \frac{(2 k+1+\gamma)}{1-\gamma}\left|b_{k}\right| \sigma_{k}\left(\alpha_{1}\right) \Omega\right\}=2(1-\gamma)\{2
\end{aligned}
$$

$$
\begin{aligned}
& -\left(\sum_{k=1}^{\infty} k \frac{(2 k-1-\gamma)}{1-\gamma}\left|a_{k}\right|+\sum_{k=1}^{\infty} k \frac{(2 k+1+\gamma)}{1-\gamma}\left|b_{k}\right|\right) \\
& \left.\cdot \sigma_{k}\left(\alpha_{1}\right) \Omega\right\} \geq 0
\end{aligned}
$$

by hypothesis in (18), which implies that $f \in \mathscr{G}_{\mathscr{H}}\left(\left[\alpha_{1}, A_{1}\right.\right.$, $\left.\left.\beta_{1}, B_{1}\right], \gamma\right)$.

Now we obtain the necessary and sufficient condition for the function $f=h+\bar{g}$ given by (16) to be in $\mathscr{T}_{\mathscr{H}}\left(\left[\alpha_{1}\right.\right.$, $\left.\left.A_{1}, \beta_{1}, B_{1}\right], \gamma\right)$.

Theorem 4. Let $f=h+\bar{g}$ be given by (16). Then $f \in$ $\mathscr{T}_{\mathscr{H}}\left(\left[\alpha_{1}, A_{1}, \beta_{1}, B_{1}\right], \gamma\right)$ if and only if

$$
\begin{aligned}
& \sum_{k=1}^{\infty} k\left\{\frac{(2 k-1-\gamma)}{1-\gamma}\left|a_{k}\right|+\frac{(2 k+1+\gamma)}{1-\gamma}\left|b_{k}\right|\right\} \sigma_{k}\left(\alpha_{1}\right) \Omega \\
& \quad \leq 2 .
\end{aligned}
$$

Proof. Since $\mathscr{T}_{\mathscr{H}}\left(\left[\alpha_{1}, A_{1}, \beta_{1}, B_{1}\right], \gamma\right) \subset \mathscr{G}_{\mathscr{H}}\left(\left[\alpha_{1}, A_{1}, \beta_{1}, B_{1}\right], \gamma\right)$, we only have to prove the necessary part of theorem. Assume that $f \in \mathscr{T}_{\mathscr{H}}\left(\left[\alpha_{1}, A_{1}, \beta_{1}, B_{1}\right], \gamma\right)$, and then by virtue of (16), we obtain

$$
\operatorname{Re}\left\{(1-\gamma)+\left(1+e^{i \phi}\right) \frac{\left[z^{2}\left(W\left[\alpha_{1}\right] h(z)\right)^{\prime \prime}+\overline{2\left(W\left[\alpha_{1}\right] g(z)\right)^{\prime}+z^{2}\left(W\left[\alpha_{1}\right] g(z)\right)^{\prime \prime}}\right]}{z\left(W\left[\alpha_{1}\right] h(z)\right)^{\prime}-\overline{z\left(W\left[\alpha_{1}\right] g(z)\right)^{\prime}}}\right\} \geq 0
$$

This is equivalent to

$$
\begin{aligned}
& \operatorname{Re}\left\{\frac{(1-\gamma) z-\left(\sum_{k=2}^{\infty} k\left[k\left(1+e^{i \phi}\right)-\gamma-e^{i \phi}\right] \sigma_{k}\left(\alpha_{1}\right) \Omega\left|a_{k}\right| z^{k}+\sum_{k=1}^{\infty} k\left[k\left(1+e^{i \phi}\right)+\gamma+e^{i \phi}\right] \sigma_{k}\left(\alpha_{1}\right) \Omega\left|b_{k}\right| \bar{z}^{k}\right)}{z-\sum_{k=2}^{\infty} k \sigma_{k}\left(\alpha_{1}\right) \Omega\left|a_{k}\right| z^{k}-\sum_{k=1}^{\infty} k \sigma_{k}\left(\alpha_{1}\right) \Omega\left|b_{k}\right| \bar{z}^{k}}\right\} \\
& =\operatorname{Re}\left\{\frac{(1-\gamma)-\sum_{k=2}^{\infty} k\left[k\left(1+e^{i \phi}\right)-\gamma-e^{i \phi}\right] \sigma_{k}\left(\alpha_{1}\right) \Omega\left|a_{k}\right| z^{k-1}-(\bar{z} / z) \sum_{k=1}^{\infty} k\left[k\left(1+e^{i \phi}\right)+\gamma+e^{i \phi}\right] \sigma_{k}\left(\alpha_{1}\right) \Omega\left|b_{k}\right| \bar{z}^{k-1}}{1-\sum_{k=2}^{\infty} k \sigma_{k}\left(\alpha_{1}\right) \Omega\left|a_{k}\right| z^{k-1}+(\bar{z} / z) \sum_{k=1}^{\infty} k \sigma_{k}\left(\alpha_{1}\right) \Omega\left|b_{k}\right| \bar{z}^{k-1}}\right\}
\end{aligned}
$$


This condition must hold for all values of $z \in E$ and for real $\phi$, so that, by taking $z=r<1$ and $\phi=0$, the above inequality reduces to

$$
\frac{(1-\gamma)-\left[\sum_{k=2}^{\infty} k(2 k-1-\gamma) \sigma_{k}\left(\alpha_{1}\right) \Omega\left|a_{k}\right| r^{k-1}+(\bar{z} / z) \sum_{k=1}^{\infty} k(2 k+1+\gamma) \sigma_{k}\left(\alpha_{1}\right) \Omega\left|b_{k}\right| r^{k-1}\right]}{1-\sum_{k=2}^{\infty} k \sigma_{k}\left(\alpha_{1}\right) \Omega\left|a_{k}\right| r^{k-1}+(\bar{z} / z) \sum_{k=1}^{\infty} k \sigma_{k}\left(\alpha_{1}\right) \Omega\left|b_{k}\right| r^{k-1}} \geq 0
$$

Letting $r \rightarrow 1^{-}$through real values, we obtain condition (22). This completes the proof.

We determine the extreme points of closed convex hulls of $\mathscr{T}_{\mathscr{H}}\left(\left[\alpha_{1}, A_{1}, \beta_{1}, B_{1}\right], \gamma\right)$ denoted by $\operatorname{clco} \mathscr{T}_{\mathscr{H}}\left(\left[\alpha_{1}\right.\right.$, $\left.\left.A_{1}, \beta_{1}, B_{1}\right], \gamma\right)$.

Theorem 5. A function $f(z) \in \operatorname{clco}_{\mathscr{H}}\left(\left[\alpha_{1}, A_{1}, \beta_{1}, B_{1}\right], \gamma\right)$ if and only if

$$
f(z)=\sum_{k=1}^{\infty}\left(X_{k} h_{k}(z)+Y_{k} g_{k}(z)\right)
$$

where

$$
\begin{aligned}
& h_{1}(z)=z, \\
& h_{k}(z)=z-\frac{1-\gamma}{k(2 k-1-\gamma) \sigma_{k}\left(\alpha_{1}\right) \Omega} z^{k} ; \quad(k \geq 2), \\
& g_{k}(z)=z-\frac{1-\gamma}{k(2 k+1+\gamma) \sigma_{k} \Omega} \bar{z}^{k} ; \quad(k \geq 2),
\end{aligned}
$$

$\sum_{k=1}^{\infty}\left(X_{k}+Y_{k}\right)=1, X_{k} \geq 0$, and $Y_{k} \geq 0$. In particular, the extreme points of $\mathscr{T}_{\mathscr{H}}\left(\left[\alpha_{1}, A_{1}, \beta_{1}, B_{1}\right], \gamma\right)$ are $\left\{h_{k}\right\}$ and $\left\{g_{k}\right\}$.

Proof. First, we consider

$$
\begin{aligned}
f(z)= & \sum_{k=1}^{\infty}\left(X_{k} h_{k}(z)+Y_{k} g_{k}(z)\right) \\
= & \sum_{k=1}^{\infty}\left(X_{k}+Y_{k}\right) z \\
& -\sum_{k=2}^{\infty} \frac{1-\gamma}{k(2 k-1-\gamma) \sigma_{k}\left(\alpha_{1}\right) \Omega} X_{k} z^{k} \\
& -\sum_{k=1}^{\infty} \frac{1-\gamma}{k(2 k+1+\gamma) \sigma_{k}\left(\alpha_{1}\right) \Omega} Y_{k} \bar{z}^{k}, \\
f(z)= & z-\sum_{k=1}^{\infty} A_{k} z^{k}-\sum_{k=1}^{\infty} B_{k} z^{k},
\end{aligned}
$$

where $A_{k}=\left((1-\gamma) / k(2 k-1-\gamma) \sigma_{k}\left(\alpha_{1}\right) \Omega\right) X_{k}$, and $B_{k}=$ $\left((1-\gamma) / k(2 k+1+\gamma) \sigma_{k}\left(\alpha_{1}\right) \Omega\right) Y_{k}$.
Using (22) for the coefficients in (29), we have

$$
\begin{aligned}
& \sum_{k=2}^{\infty} \frac{k(2 k-1-\gamma) \sigma_{k}\left(\alpha_{1}\right) \Omega}{1-\gamma} A_{k} \\
& \quad+\sum_{k=1}^{\infty} \frac{k(2 k+1+\gamma) \sigma_{k}\left(\alpha_{1}\right) \Omega}{1-\gamma} B_{k}=\sum_{k=2}^{\infty} X_{k}+\sum_{k=1}^{\infty} Y_{k} \\
& \quad=1-x_{1} \leq 1,
\end{aligned}
$$

and hence $f(z) \in \operatorname{clco} \mathscr{T}_{\mathscr{H}}\left(\left[\alpha_{1}, A_{1}, \beta_{1}, B_{1}\right], \gamma\right)$.

Conversely, suppose that $f(z) \in \operatorname{clco} \mathscr{T}_{\mathscr{H}}\left(\left[\alpha_{1}, A_{1}\right.\right.$, $\left.\left.\beta_{1}, B_{1}\right], \gamma\right)$, and set

$$
\begin{aligned}
& X_{k}=\frac{k(2 k-1-\gamma) \sigma_{k}\left(\alpha_{1}\right) \Omega}{1-\gamma} A_{k}, \quad(k \geq 2), \\
& Y_{k}=\frac{k(2 k+1+\gamma) \sigma_{k}\left(\alpha_{1}\right) \Omega}{1-\gamma} B_{k}, \quad(k \geq 1),
\end{aligned}
$$

where $\sum_{k=1}^{\infty}\left(X_{k}+Y_{k}\right)=1$. Then

$$
\begin{aligned}
f(z)= & z-\sum_{k=2}^{\infty} A_{k} z^{k}-\sum_{k=1}^{\infty} B_{k} \bar{z}^{k}, \quad A_{k} \geq 0, B_{k} \geq 0 \\
= & z-\sum_{k=2}^{\infty} \frac{1-\gamma}{k(2 k-1-\gamma) \sigma_{k}\left(\alpha_{1}\right) \Omega} X_{k} z^{k} \\
& -\sum_{k=1}^{\infty} \frac{1-\gamma}{k(2 k+1+\gamma) \sigma_{k}\left(\alpha_{1}\right) \Omega} Y_{k} \bar{z}^{k} \\
= & z+\sum_{k=2}^{\infty}\left(h_{k}(z)-z\right) X_{k}+\sum_{k=1}^{\infty}\left(g_{k}(z)-z\right)_{k} Y_{k} \\
= & \sum_{k=1}^{\infty}\left(X_{k} h_{k}(z)+Y_{k} g_{k}(z)\right)
\end{aligned}
$$

which is the required result.

Next we show that $\mathscr{T}_{\mathscr{C}}\left(\left[\alpha_{1}, A_{1}, \beta_{1}, B_{1}\right], \gamma\right)$ is close under convex combinations of its members.

Theorem 6. The family $\mathscr{T}_{\mathscr{H}}\left(\left[\alpha_{1}, A_{1}, \beta_{1}, B_{1}\right], \gamma\right)$ is closed under convex combination.

Proof. For $i=1,2, \ldots$, suppose that $f_{i} \in \mathscr{T}_{\mathscr{H}}\left(\left[\alpha_{1}, A_{1}\right.\right.$, $\left.\left.\beta_{1}, B_{1}\right], \gamma\right)$, where

$$
f_{i}(z)=z-\sum_{k=2}^{\infty} a_{i, k} z^{k}-\overline{\sum_{k=2}^{\infty} b_{i, k} z^{k}}
$$


For coefficients in (22) the relation given in (33) takes the form

$$
\sum_{k=2}^{\infty} \frac{k(2 k-1-\gamma) \sigma_{k} \Omega}{1-\gamma} a_{i, k}+\sum_{k=1}^{\infty} \frac{k(2 k+1+\gamma) \sigma_{k} \Omega}{1-\gamma} b_{i, k}
$$

$\leq 1$.

For $\sum_{i=1}^{\infty} t_{i}=1,0 \leq t_{i} \leq 1$, the convex combination of $f_{i}$ may be written as

$$
\sum_{i=1}^{\infty} t_{i} f_{i}(z)=z-\sum_{k=2}^{\infty}\left(\sum_{i=1}^{\infty} t_{i} a_{i, k}\right) z^{k}-\overline{\sum_{k=2}^{\infty}\left(\sum_{i=1}^{\infty} t_{i} b_{i, k}\right)} z^{k}
$$

From inequality (22) for (35),

$$
\begin{aligned}
& \sum_{k=2}^{\infty} \frac{k(2 k-1-\gamma) \sigma_{k}\left(\alpha_{1}\right) \Omega}{1-\gamma}\left(\sum_{i=1}^{\infty} t_{i} a_{i, k}\right) \\
& +\sum_{k=1}^{\infty} \frac{k(2 k+1+\gamma) \sigma_{k}\left(\alpha_{1}\right) \Omega}{1-\gamma}\left(\sum_{i=1}^{\infty} t_{i} a_{i, k}\right) \\
& \quad \cdot \sum_{i=1}^{\infty} t_{i}\left(\sum_{k=2}^{\infty} \frac{k(2 k-1-\gamma) \sigma_{k}\left(\alpha_{1}\right) \Omega}{1-\gamma} a_{i, k}\right. \\
& \left.+\sum_{k=1}^{\infty} \frac{k(2 k+1+\gamma) \sigma_{k}\left(\alpha_{1}\right) \Omega}{1-\gamma} b_{i, k}\right) \leq \sum_{t=1}^{\infty} t_{i}=1,
\end{aligned}
$$

and therefore $\sum_{t=1}^{\infty} t_{i} f_{i}(z) \in \mathscr{T}_{\mathscr{H}}\left(\left[\alpha_{1}, A_{1}, \beta_{1}, B_{1}\right], \gamma\right)$.

Theorem 7. For $0 \leq \delta \leq \gamma \leq 1$, let $f(z) \in$ $\mathscr{T}_{\mathscr{H}}\left(\left[\alpha_{1}, A_{1}, \beta_{1}, B_{1}\right], \gamma\right)$ and $F(z) \in \mathscr{T}_{\mathscr{H}}\left(\left[\alpha_{1}, A_{1}, \beta_{1}, B_{1}\right], \delta\right)$. Then

$$
\begin{aligned}
& f(z) * F(z) \in \mathscr{T}_{\mathscr{H}}\left(\left[\alpha_{1}, A_{1}, \beta_{1}, B_{1}\right], \gamma\right) \\
& \subset \mathscr{T}_{\mathscr{H}}\left(\left[\alpha_{1}, A_{1}, \beta_{1}, B_{1}\right], \delta\right) .
\end{aligned}
$$

Proof. Let

$$
\begin{aligned}
f(z) & =z-\sum_{k=2}^{\infty} a_{k} z^{k}-\overline{\sum_{k=1}^{\infty} b_{k} z^{k}} \\
& \in \mathscr{T}_{\mathscr{H}}\left(\left[\alpha_{1}, A_{1}, \beta_{1}, B_{1}\right], \gamma\right), \\
F(z) & =z-\sum_{k=2}^{\infty} A_{k} z^{k}-\overline{\sum_{k=1}^{\infty} B_{k} z^{k}} \\
& \in \mathscr{T}_{\mathscr{H}}\left(\left[\alpha_{1}, A_{1}, \beta_{1}, B_{1}\right], \delta\right),
\end{aligned}
$$

and then

$$
f(z) * F(z)=z+\sum_{k=2}^{\infty} a_{k} A_{k} z^{k}+\overline{\sum_{k=1}^{\infty} b_{k} B_{k} z^{k}}
$$

We note that $\left|A_{k}\right| \leq 1$ and $\left|B_{k}\right| \leq 1$. Therefore

$$
\begin{aligned}
\sum_{k=2}^{\infty} & \frac{k(2 k-1-\delta) \sigma_{k}\left(\alpha_{1}\right) \Omega}{1-\delta}\left|a_{k}\right|\left|A_{k}\right| \\
& +\sum_{k=1}^{\infty} \frac{k(2 k+1+\delta) \sigma_{k}\left(\alpha_{1}\right) \Omega}{1-\delta}\left|b_{k}\right|\left|B_{k}\right| \\
& \leq \sum_{k=2}^{\infty} \frac{k(2 k-1-\delta) \sigma_{k}\left(\alpha_{1}\right) \Omega}{1-\delta}\left|a_{k}\right| \\
& +\sum_{k=1}^{\infty} \frac{k(2 k+1+\delta) \sigma_{k}\left(\alpha_{1}\right) \Omega}{1-\delta} b_{k} \\
& \leq \sum_{k=2}^{\infty} \frac{k(2 k-1-\gamma) \sigma_{k}\left(\alpha_{1}\right) \Omega}{1-\gamma}\left|a_{k}\right| \\
& +\sum_{k=1}^{\infty} \frac{k(2 k+1+\gamma) \sigma_{k}\left(\alpha_{1}\right) \Omega}{1-\gamma}\left|b_{k}\right| \leq 1,
\end{aligned}
$$

by using (22), since $f(z) \in \mathscr{T}_{\mathscr{H}}\left(\left[\alpha_{1}, A_{1}, \beta_{1}, B_{1}\right], \gamma\right)$ and $0 \leq$ $\delta \leq \gamma \leq 1$. This proves that $f(z) * F(z) \in \mathscr{T}_{\mathscr{H}}\left(\left[\alpha_{1}\right.\right.$, $\left.\left.A_{1}, \beta_{1}, B_{1}\right], \delta\right)$.

Now for the class $\mathscr{T}_{\mathscr{H}}\left(\left[\alpha_{1}, A_{1}, \beta_{1}, B_{1}\right], \gamma\right)$ the closure property under the generalized Bernardi-Livingston integral operator $L_{c}(f)$ is examined which is defined by

$$
L_{c}(f)=\frac{c+1}{z^{c}} \int_{0}^{z} t^{c-1} f(t) d t, \quad c>-1 .
$$

Theorem 8. Let $f(z) \in \mathscr{T}_{\mathscr{H}}\left(\left[\alpha_{1}, A_{1}, \beta_{1}, B_{1}\right], \gamma\right)$ and then $L_{c}(f) \in \mathscr{T}_{\mathscr{H}}\left(\left[\alpha_{1}, A_{1}, \beta_{1}, B_{1}\right], \gamma\right)$.

Proof. Consider the generalized Bernardi-Livingston integral operator $L_{c}(f)$ given in (41):

$$
\begin{aligned}
& L_{c}(f)=\frac{c+1}{z^{c}} \int_{0}^{z} t^{c-1}[h(t)+\overline{g(t)}] d t \\
& =\frac{c+1}{z^{c}}\left(\int_{0}^{z} t^{c-1}\left(t-\sum_{k=2}^{\infty} a_{k} t^{k}\right) d t\right. \\
& \left.\quad-\overline{\int_{0}^{z} t^{c-1}\left(\sum_{k=1}^{\infty} b_{k} t^{k}\right)}\right) \\
& =z-\sum_{k=2}^{\infty} A_{k} z^{k}-\overline{\sum_{k=1}^{\infty} B_{k} z^{k}},
\end{aligned}
$$

where

$$
\begin{aligned}
A_{k} & =\frac{c+1}{c+n} a_{k} ; \\
B_{k} & =\frac{c+1}{c+n} b_{k} .
\end{aligned}
$$


Therefore

$$
\begin{aligned}
\sum_{k=1}^{\infty} k & \left(\frac{(2 k-1-\gamma)}{1-\gamma}\left(\frac{c+1}{c+n}\right)\left|a_{k}\right|\right. \\
+ & \left.\frac{(2 k+1+\gamma)}{1-\gamma}\left(\frac{c+1}{c+n}\right)\left|b_{k}\right|\right) \sigma_{k}\left(\alpha_{1}\right) \Omega \\
\leq & \sum_{k=1}^{\infty} k\left(\frac{(2 k-1-\gamma)}{1-\gamma}\left|a_{k}\right|+\frac{(2 k+1+\gamma)}{1-\gamma}\left|b_{k}\right|\right) \\
\cdot & \sigma_{k}\left(\alpha_{1}\right) \Omega \leq 2(1-\gamma) .
\end{aligned}
$$

Since $f(z) \in \mathscr{T}_{\mathscr{H}}\left(\left[\alpha_{1}, A_{1}, \beta_{1}, B_{1}\right], \gamma\right)$, therefore, by Theorem $4, L_{c}(f) \in \mathscr{T}_{\mathscr{H}}\left(\left[\alpha_{1}, A_{1}, \beta_{1}, B_{1}\right], \gamma\right)$.

\section{Competing Interests}

The authors declare that they have no competing interests.

\section{Acknowledgments}

This work is supported by AP-2013-009 and GP-K006392, Universiti Kebangsaan Malaysia.

\section{References}

[1] E. Heinz, Über die Lösungen der Minimalflächengleichung, von Erhard Heinz, Vandenhoeck und Ruprecht, Göttingen, Germany, 1952.

[2] A. Weitsman, "On univalent harmonic mappings and minimal surfaces," Pacific Journal of Mathematics, vol. 192, no. 1, pp. 191200, 2000.

[3] J. Clunie and T. Sheil-Small, "Harmonic univalent functions," Annales Academiae Scientiarum Fennicae A, vol. 9, pp. 3-25, 1984.

[4] J. M. Jahangiri, "Harmonic functions starlike in unit dics," Journal of Mathematical Analysis and Applications, vol. 235, no. 2, pp. 470-477, 1999.

[5] H. Silverman and E. M. Silvia, "Subclasses of harmonic univalent functions," The New Zealand Journal of Mathematics, vol. 28, no. 2, pp. 275-284, 1999.

[6] H. Silverman, "Harmonic univalent functions with negative coefficients," Journal of Mathematical Analysis and Applications, vol. 220, no. 1, pp. 283-289, 1998.

[7] E. M. Wright, "The asymptotic expansion of the generalized hypergeometric function," Proceedings of the London Mathematical Society, vol. 46, pp. 389-408, 1946.

[8] J. Dziok and H. M. Srivastava, "Certain subclasses of analytic functions associated with the generalized hypergeometric function," Integral Transforms and Special Functions, vol. 14, no. 1, pp. 7-18, 2003.

[9] J. Dzoik and H. M. Srivastava, "Class of analytic associated with generalized hypergeometric function," Applied Mathematics and Computation, vol. 103, pp. 1-13, 1999.

[10] J. Dzoik and H. M. Srivastava, "Some subclasses of analytic functions with fixed argument of coefficients associated with the generalized hypergeometric function," Advanced Studies in Contemporary Mathematics, vol. 5, no. 2, pp. 115-125, 2002.
[11] B. C. Carlson and D. B. Shaffer, "Starlike and prestarlike hypergeometric functions," SIAM Journal on Mathematical Analysis, vol. 15, no. 4, pp. 737-745, 1984.

[12] S. D. Bernardi, "Convex and starlike univalent functions," Transactions of the American Mathematical Society, vol. 135, pp. 429-446, 1969.

[13] J. Dziok and R. K. Raina, "Families of analytic functions associated with the Wright generalized hypergeometric function," Demonstratio Mathematica, vol. 37, no. 3, pp. 533-542, 2004.

[14] O. P. Ahuja, S. Nagpal, and V. Ravichandran, "Radius constants for functions with the prescribed coefficient bounds," Abstract and Applied Analysis, vol. 2014, Article ID 454152, 12 pages, 2014.

[15] Y. Avci and E. Zlotkiewicz, "On harmonic univalent mappings," Maria Curie-Skłodowska University, vol. 44, pp. 1-7, 1990.

[16] J. M. Jahangiri, "Coefficient bounds and univalance criteria for harmonic functions with negative coefficent," Annales Academiae Scientiarum Fennicae A, pp. 57-66, 1998.

[17] J. M. Jahangiri, G. Murugusundaramoorthy, and K. Vijaya, "Starlikeness of Rucheweyh type harmonic univalent functions," The Journal of the Indian Academy of Mathematics, vol. 26, no. 1, pp. 191-200, 2004.

[18] S. B. Joshi and M. Darus, "Uni.ed treatment for harmonic univalent functions," Tamsui Oxford Journal of Information and Mathematical Sciences, vol. 24, no. 3, pp. 225-232, 2008.

[19] G. Murugusundaramoorthy, "A class of Ruscheweyh-type harmonic univalent functions with varying arguments," Southwest Journal of Pure and Applied Mathematics, vol. 2, pp. 90-95, 2003.

[20] T. Rosy, B. A. Stephen, K. G. Subramanian, and J. M. Jhangiri, "Goodman harmonic convex functions," Journal of Natural Geometry, vol. 21, no. 1-2, pp. 39-50, 2001.

[21] J. Sokół, R. W. Ibrahim, M. Z. Ahmad, and H. F. Al-Janaby, "Inequalities of harmonic univalent functions with connections of hypergeometric functions," Open Mathematics, vol. 13, no. 1, pp. 691-705, 2015.

[22] R. Chandrashekar, G. Murugusundaramoorthy, S. K. Lee, and K. G. Subramanian, "A class of complex valued harmonic functions de.ned by Dzoik Srivastava operator," Chamchuri Journal of Mathematics, vol. 1, no. 2, pp. 31-42, 2009. 


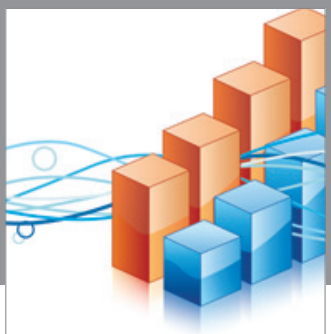

Advances in

Operations Research

vatem alat4

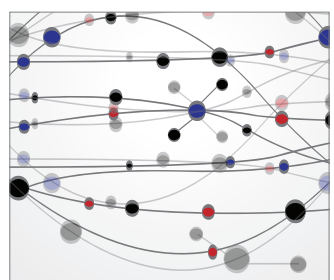

\section{The Scientific} World Journal
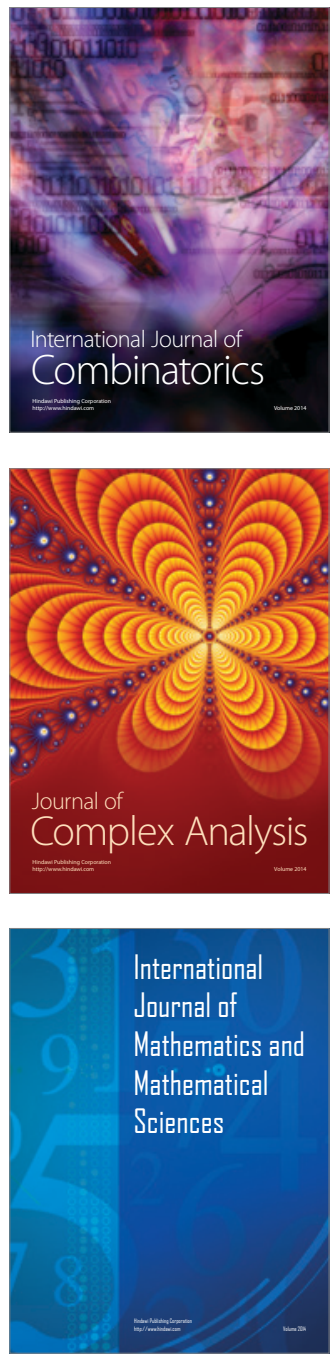
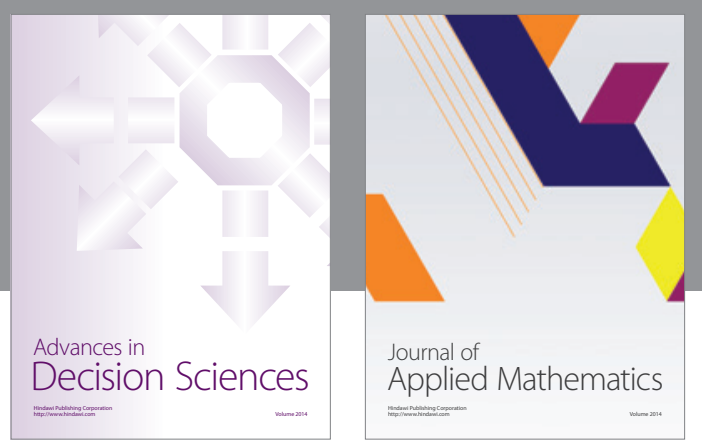

Algebra

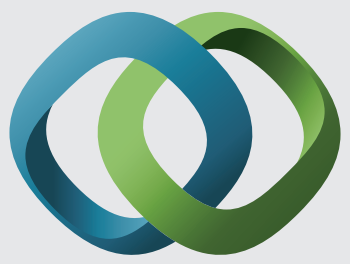

\section{Hindawi}

Submit your manuscripts at

http://www.hindawi.com
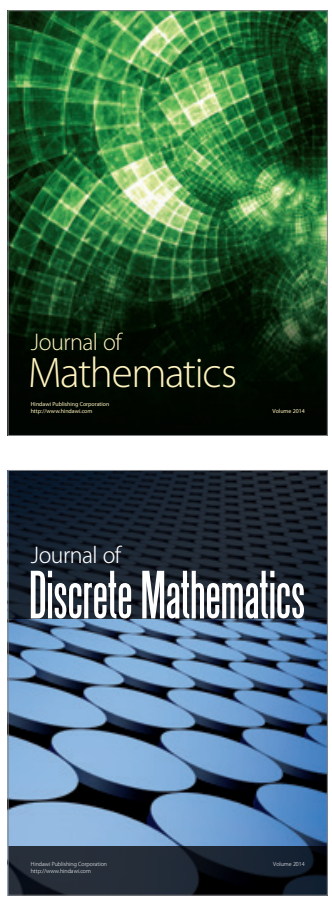

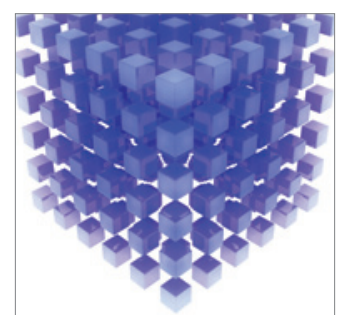

Mathematical Problems in Engineering
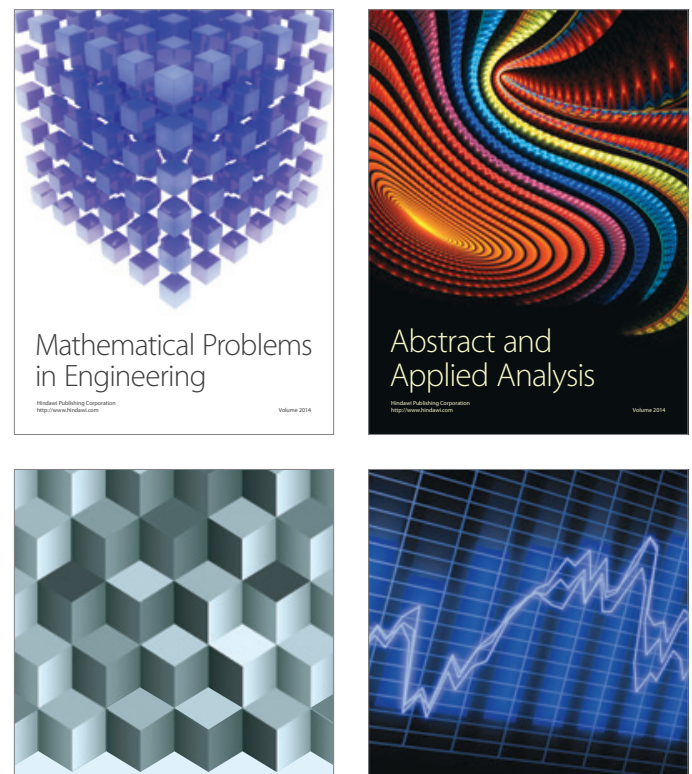

Journal of

Function Spaces

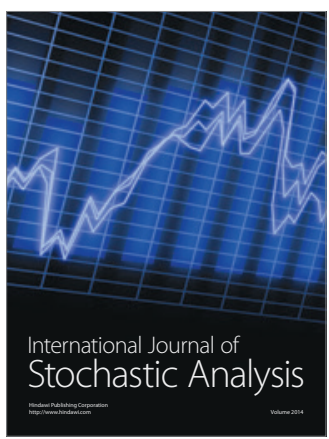

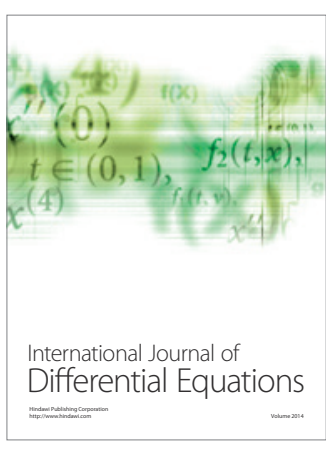
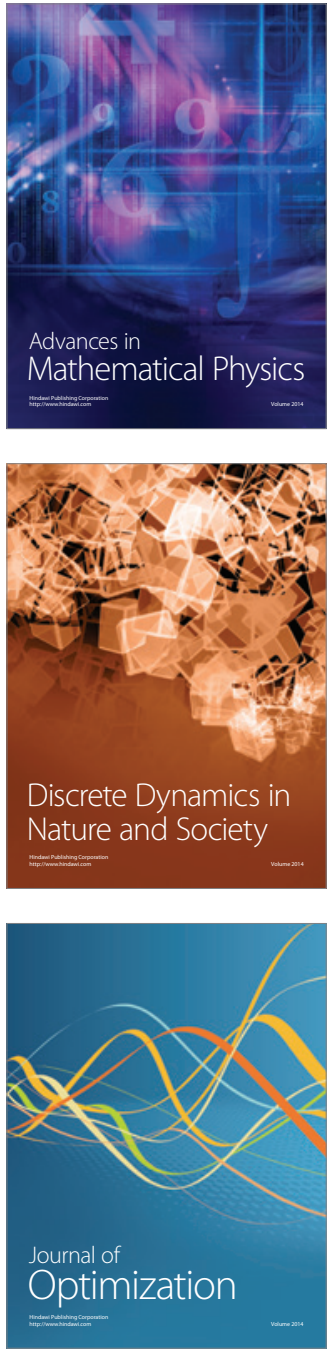